Horizons philosophiques

\title{
Hannah Arendt : Erôs de la liberté de penser et amour de la liberté politique
}

\section{Francis Moreault}

Volume 11, numéro 2, printemps 2001

L’Europe, le laboratoire d'idées du XXI ${ }^{\mathrm{e}}$ siècle?

URI : https://id.erudit.org/iderudit/801185ar

DOI : https://doi.org/10.7202/801185ar

Aller au sommaire du numéro

Éditeur(s)

Collège Édouard-Montpetit

ISSN

1181-9227 (imprimé)

1920-2954 (numérique)

Découvrir la revue

\section{Citer cet article}

Moreault, F. (2001). Hannah Arendt : Erôs de la liberté de penser et amour de la liberté politique. Horizons philosophiques, 11(2), 109-129.

https://doi.org/10.7202/801185ar d'utilisation que vous pouvez consulter en ligne.

https://apropos.erudit.org/fr/usagers/politique-dutilisation/ 


\section{HANNAH ARENDT : ERÔS DE LA LIBERTÉ DE PENSERET AMOUR DE LA LIBERTÉ POLITIQUE}

D'aucuns seront sans doute surpris de voir ici examiner le concept de l'amour dans la pensée politique de Hannah Arendt. Car l'amour n'est-il pas la passion par excellence qui doit être strictement réservée au domaine de la maisonnée, de l'oikos, bref au domaine privé chez elle? Est-ce qu'il n'est pas déjà entendu que l'amour ne concerne nullement l'espace du politique? Pour un écrivain politique qui s'est toujours efforcé de séparer la vie privée de la vie publique, l'idion du koinon, il est certainement saugrenu d'associer ici l'amour avec la politique. Pourtant, une analyse minutieuse du concept de l'amour dans la théorie politique d'Arendt nous démontre que cette notion n'est pas aussi étrangère qu'une première lecture nous le laisserait croire, à sa compréhension du politique. La philosophe allemande développe, nous semble-t-il, une double théorie de l'amour dans son rapport avec la politique : l'amour (l'erôs) de la liberté de penser et l'amour de la liberté politique. En ce qui concerne la première théorie, il faut d'abord saisir en quoi consiste l'activité de penser selon Arendt. Nous devons ensuite examiner le rapport qu'elle tisse entre l'amour et cette activité réflexive. Enfin, en quel sens l'activité de penser est-elle libre chez elle? À l'égard de la seconde théorie, on doit examiner la relation qu'établit Arendt entre l'amour et la liberté politique. Pourquoi associe-t-elle l'amour avec la liberté d'agir? En quel sens, en second lieu, l'amour de la liberté politique est-il un principe de l'action? Pourquoi sommes-nous, en définitive, "condamnés à la liberté»? Enfin, est-ce que l'amour est un concept radicalement anti-politique dans la pensée d'Arendt? Autrement dit, faut-il se contenter de confiner l'amour arendtien dans l'espace de l'oikos, de la maisonnée?

\section{L'amour est-il anti-politique?}

Commençons par cette dernière question. Arendt conçoit certes, au premier abord, l'amour comme une relation fondée sur la passion. II ne s'adresse pas ainsi à des entités abstraites comme l'amour d'un peuple ou l'amour d'un pays, mais uniquement à "des personnes 1 ". Dans ce

1. H. ARENDT et G. SCHOLEM, «Le procès Eichmann: un débat avec Hannah Arendt", dans Fidélité et utopie. Essais sur lejudaïsme contemporain, Paris, Calmann-Lévy, 1978, p. 223. 
sens, l'amour est étranger au domaine public, à la sphère d'apparence. II “(...) s'éteint dès que l'on en fait étalage. ( "Ne parle pas de ton amour. De l'amour qui ne se peut jamais dire»). Essentiellement étranger au monde, l'amour ne peut que mentir et se pervertir lorsqu'on l'emploie à des fins politiques comme le changement ou le salut du monde2». II doit être indubitablement caché et ne pas apparaître sur la place publique; il est donc anti-politique ${ }^{3}$. Mais comme le remarque Françoise Collin ${ }^{4}$, l'amour arendtien est dans le domaine privé ce que le respect est dans le domaine public. L'amour révèle, en effet, le «qui» du sujet amoureux, lidentité de l'individu amoureux dans sa relation avec l'être aimé. Toutefois, la professeure de théorie politique est très brève sur ce point. C'est parce qu'il est désintéressé, soutient-elle laconiquement, que l'homme révèle ce qu'il est, dévoile son visage à l'être aimé. L'amour est en quelque sorte une forme de respect de l'autre, une forme "d'amitié sans intimité, sans proximités ». II correspond donc à une forme d'amitié aristotélicienne dans la mesure où l'amour met une distance entre les amoureux dans laquelle s'insère le monde. Bien que l'amour tende à détruire l'inter homines esse, il trouve cependant sa corrélation dans le respect d'autrui et dans l'amitié qui sont, bien sûr, des conditions pour exercer l'action politique. D'autre part, l'amour éloigne certes les amoureux du domaine public, mais ceux-ci réintègrent d'une certaine manière non seulement ce domaine par le respect mutuel, mais aussi par le produit de l'union de leur amour, c'est-àdire l'enfant. En insérant le nouveau venu dans le monde, les parents pénètrent ainsi à nouveau dans ce dernier. S'identifiant au respect et à l'amitié aristotélicienne, l'amour a une dimension politique dans le sens où, d'une part, il révèle l'identité de l'être amoureux et dans le sens où, d'autre part, il maintient un intervalle entre l'amant et l'amante dans lequel s'introduit le monde. D'emblée, l'amour-passion a, dans une certaine mesure, une connotation politique chez Arendt. On ne peut donc réduire cet amour arendtien, comme le fait George Kateb, à sa seule et unique dimension anti-politique 6 .

2. H. ARENDT, Condition de l'homme modeme, Paris, Presses Pocket, 1983, p. 91.

3. Ibid., p. 309. Selon M.-L. B. de Launay et A. Enegrén, les deux vers cités par Arendt sont tirés d'un poème de Blake qui figure dans le Manuscrit Rosetti. Voir $\mathrm{H}$. ARENDT, "Le grand jeu du mondew, dans Esprit, no 1667 (juin 1985), p.182, note 7.

4. F. COLLIN, "Du privé et du public", dans Les Cahiers du GRIF, no 33 (printemps 1986), p. 53-54.

5. H. ARENDT, Condition de l'homme modeme, p. 308.

6. G. KATEB, "Freedom and Worldliness in the Thought of Hannah Arendt", dans Political Theory, vol. 5, no 2 (mai 1977), p. 161. Repris dans G. KATEB, Hannah Arendt : Politics, Conscience, Evil, New Jersey, Rourman and Allanheld, 1984. 


\section{L'erôs ou comment exercer l'activité de penser}

Refusant d'identifier l'amour uniquement à la passion, Arendt nous invite ainsi à poursuivre la réflexion sur le concept de l'amour. Dans ce sens, il faut examiner le lien qu'elle établit entre ce concept et l'activité de penser de Socrate. Arendt nous sert ici de guide : elle précise, dans son entretien avec Gauss, que "ce qui importe, c'est le processus de pensée lui-même7". Qu'est-ce à dire? Qu'est-ce qu'elle entend par "processus de pensée"? Or, ce processus de pensée s'inspire grandement - il nous semble - de l'activité de penser socratique. Quelle est donc la vision socratique de l'activité de penser? Pour Arendt, celle-ci est fondée sur trois caractères. En premier lieu, le processus de pensée de Socrate est engendré par le "deux-en-un» - le "dialogue intérieur dans lequel on converse avec soi-même ${ }^{8}$ ». C'est l'expérience de la différence au sein de la consciousness, c'est-à-dire je suis à la fois celui qui pose une question et celui qui y répond, qui fonde le processus de pensée chez Socrate. Or, c'est "cette dualité de moi face à moi-même qui fait de la pensée une activité véritable" ". C'est pourquoi Arendt écrit "l'activité de penser" de Socrate et non pas l'activité de la pensée de Socrate parce qu'elle veut souligner le caractère actif du penser de ce dernier. Ce penser est actif dans le sens où il implique un dialogue constant entre moi et moi-même. Mais pour que cette dualité intérieure s'exerce, il faut que la relation entre le "premier" moi et le "second" moi soit harmonieuse. Dans le dialogue pensant de Socrate, l'autre moi-même est donc en quelque sorte un ami. En faisant l'expérience de l'amitié au niveau de l'esprit, l'activité de penser de Socrate est ainsi pour Arendt conditionnée par la pluralité. Or, la pluralité est non seulement et précisément la condition de l'activité de penser mais aussi la condition de l'action chez elle. Au sein de la vie de l'esprit, le deux-en-un socratique fonde donc un être ensemble. C'est pourquoi elle reprend ce caractère de l'activité de penser socratique.

Le deux-en-un socratique engendre, en deuxième lieu, un mouvement de pensée, c'est-à-dire il entreprend une quête qui vise à saisir le sens d'un mot ou d'un concept. Plus précisément, ce chemin de pensée consiste à dégeler, à défaire les définitions, les concepts légués par la tradition pour comprendre le sens original de ces derniers ${ }^{10}$. La vie de l'esprit de Socrate procède ainsi à un examen critique des concepts constitutifs de notre tradition de pensée. Elle ne produit aucune définition, ne

7. H. ARENDT, "Seule demeure la langue maternelle", dans La tradition cachée : le Juif comme paria, Paris, Bourgois, 1987, p. 225.

8. H. ARENDT, Condition de l'homme modeme, p. 365.

9. H. ARENDT, La vie de l'esprit 1 : La pensée, Paris, PUF, 1981, p. 210.

10. Ibid., 196. 
parvient pas à connaître un objet de pensée; elle ne fait, pour employer une formule à la mode, que "déconstruire" les concepts"1. Cette quête de sens qui consiste à déconstruire les concepts, Socrate la nomme méditation. II s'agit non pas de définir un concept mais plutôt de méditer le sens de ce dernier. Dès lors que Socrate s'efforce de définir un mot, il se dérobe à lui. Par conséquent, l'activité de penser socratique n'engendre pas de "stabilité"; elle laisse tout en mouvement. C'est la raison pour laquelle cette activité souffle, pour reprendre le mot d'Arendt, comme le "vent" (elle prend même parfois la forme d'un "orage" (Heidegger). Ce deux-en-un balaye tout sur son passage et ne laisse rien derrière lui. Penser, c'est comme tisser la toile de Pénélope : "Elle défait chaque matin ce qu'elle a fait la nuit précédente ${ }^{12}$ ". On doit donc toujours repenser ce qui a déjà été pensé.

Méditatif et incapable d'engendrer de la stabilité, le deux-en-un socratique ne nous laisse, par conséquent et en troisième lieu, que devant des "incertitudes", des perplexités. II nous conduit à des apories, c'est-à-dire il ne nous mène nulle part. Socrate ne peut connaître ce que c'est, par exemple, la justice ou la sagesse, il peut seulement y penser. Penser avec Socrate, c'est donc ufaire l'expérience réelle de nonsavoir ${ }^{13}$ ". Or, ce processus de pensée socratique fondé, selon la philosophe allemande, sur le deux-en-un - l'expérience de l'altérité au sein de mon unicité, s'exerçant selon le procédé de l'examen critique déconstructif qui mène n'importe où, à un état de scepticisme, Socrate le nomme erôs, amour. Autrement dit, cette quête de signification qui fraie le chemin de pensée socratique est en quelque sorte un acte amoureux ${ }^{14}$. Pour comprendre cette thèse d'Arendt à savoir que nous pensons parce que nous sommes amoureux du questionnement, du thaumadzein, il faut, bien entendu, connaître ce que Socrate désigne par ce thème d'erôs? Qu'est-ce que l'erôs socratique?

Platon développe principalement le thème de l'amour dans un de ses dialogues : Le Banquet. Nous savons que, dans ce dialogue, des Grecs dont Socrate se réunissent autour d'un repas et font l'éloge de l'amour. Chacun des Grecs prend tour à tour la parole. Puis vient le tour de Socrate. II souligne d'abord que l'amour se définit par l'absence. On désire ce que l'on ne possède pas : "L'homme comme tous ceux qui désirent, désire ce qui n'est pas actuel ni présent; ce qu'on n'a pas, ce qu'on n'est pas, ce dont on manque, voilà les objets du désir et de

11. H. ARENDT, «Philosophie et politique", dans Les Cahiers du GRIF, p. 91.

12. H. ARENDT, La vie de l'esprit 1 : La pensée, p. 105.

13. H. ARENDT, "Philosophie et politique", p. 91.

14. H. ARENDT, La vie de l'esprit 1 : La pensée, p. 203. 
l'amour15». L'amour ne signifie donc pas pour Socrate l'unité retrouvée. II s'oppose ici à Aristophane selon lequel l'amour consiste "à se réunir et à se fondre avec l'objet aimé et à ne plus faire qu'un au lieu de deux ${ }^{16}$ ". En revanche, la réalisation de l'unité, la fusion entre les deux êtres met un terme pour Socrate à l'amour. II y a donc un acte amoureux, un désir amoureux chez Socrate. Car l'homme est toujours à la recherche de quelque chose qu'il n'a pas. Or, nous avons vu que le processus de pensée d'Arendt consiste à poursuivre une quête de signification qui mène nulle part, à des apories. Hannah Arendt, cette amoureuse, pour reprendre son expression, des "perplexités" ajoute ici : en désirant ce qu'il n'a pas, l'amour établit des liens avec "ce qui n'est pas présent17». C'est donc la quête de ce qui nous manque qui la conduit aussi à entreprendre ce chemin de pensée. Elle suit donc ici Socrate. Chez elle, c'est l'amour au sens socratique (erôs) qui établit au niveau de la consciousness le lien entre l'objet désiré et le désir, entre le monde des apparences et le monde invisible. Par l'amour, je me représente dans le deux-en-un, «l'actualisation de la différence présente dans la consciousness" ce qui est absent. Cette absence invite Arendt à réfléchir comment fonder l'activité de penser. Elle y parvient ici par l'amour socratique : «Seuls ceux qu'inspire l'erôs socratique, l'amour de la sagesse sont capables de penser et sont dignes de confiance ${ }^{18}$ \%. Le processus de l'activité arendtienne de penser est ainsi fondé sur l'amour. II est donc la condition a priori - le "prerequisit» - à l'exercice de penser. Arendt est conduite à exercer l'activité de penser parce qu'elle est amoureuse de la "sagesse" et qu'elle tient à soumettre les objets de pensée à un examen critique. De sorte que les objets de pensée sont des objets aimables, c'est-à-dire si la quête de sens est une sorte de quête amoureuse, les objets de pensée sont donc d'une certaine façon des "choses aimables". Les objets de pensée comme la justice, la liberté et l'autorité sont des "choses aimables" parce qu'elles sont constitutives de la fondation d'un monde commun. Bref, seul l'amour de la pensée, le désir de penser à ce qui nous manque, conduit donc la théoricienne du politique à penser ce que nous faisons ${ }^{19}$.

15. PLATON, Le Banquet (trad. et éd. P. Vicaire), t. IV, $2 e$ partie, Paris, Les Belles Lettres, 1989, $200 \mathrm{~b}$.

16. Ibid., 192 e. Aristophane est d'accord cependant avec Socrate pour soutenir que l'amour est un désir (193 a).

17. H. ARENDT, Considérations morales, Paris, Payot, 1996, p. 57.

18. H. ARENDT, La vie de l'esprit 1 : La pensée, p. 205.

19. Sur le rapport entre la pensée et l'erós socratique, il faut noter qu'Arendt a bénéficié sans aucun doute du cours qu'a enseigné son second mari, Heinrich Blücher, au Bard College dans les années 1950 : aMisery and Grandeur of the Metaphysical Eros. On the Concept of philosophical Loven. Le manuscrit de ce cours n'est pas disponible, mais la publication récente, en revanche, du cours de Blücher, 
Quête infinie du désir, de ce qui nous manque, l'amour socratique rappelle-t-il l'amour augustin20? Certes, le premier et le second sont fondés sur le désir. Mais, le désir augustin est différent de celui de Socrate. Le premier consiste à rechercher la possession d'un bien en vue de mener une vie heureuse, tandis que le second, nous l'avons vu, consiste à entreprendre une quête réflexive dans le but de comprendre un objet de pensée. Le premier concerne, en un mot, la vie (au sens d'Arendt) alors que le second concerne l'activité de penser. On ne saurait davantage confondre l'erôs socratique avec l'amour chrétien (agape). Celui-ci désigne l'amour de Dieu, c'est-à-dire l'amour pour le salut chrétien d'une vie réconciliée éternellement avec ce dernier21. C'est un amour transcendant qui nous conduit au-delà de l'immanence. Or, il n'y a pas d'amour "magique", entendu comme technique de salut pour un monde "enchanté» (le royaume des cieux) chez Socrate22.

Si l'amour socratique ne permet pas de connaître l'objet de pensée, pourquoi faut-il alors penser? Puisque l'activité de penser ne mène nulle part, pourquoi penser? II n'est pas futile de se poser des questions, car même si nous échouons à définir un concept, l'exercice même de l'activité nous rend apte à comprendre un objet de pensée. On devient toujours "meilleur" en se posant des questions. Or, Socrate défend également, selon Arendt, cette même thèse : «Assez curieusement, c'est aussi l'opinion de Socrate : bien que nous ne puissions définir ni la justice ni la piété ni la bonté, et que nous ayons de la difficulté à persuader les hommes en ces matières, nous devenons plus justes ou plus pieux en y pensant et en en parlant ${ }^{23}$,. II n'est donc pas totalement futile de penser. Dans d'autres textes, Arendt va encore plus loin : un individu qui occulte la faculté de penser nie une dimension de la condition humaine. Elle écrit : "Une vie sans pensée n'a rien d'impossible; elle ne réussit pas (cependant) à développer sa propre essence - elle n'est pas seulement dépourvue de signification; elle n'est pas tout à fait vivante. Les hommes qui ne pensent

"Common Coursen, nous indique que le concept de l'erós socratique était très familier au second mari d'Arendt. Pour le cours sur l'Éros, voir dans la Correspondance Hannah Arendt-Heinrich Blücher 1936-1968, Paris, Calmann-Lévy, 1999, la lettre datée du 17 mai 1952, p. 242-243, note 1. Sur le "Common Course", voir aussi dans la correspondance les pages 519-530.

20. H. ARENDT, Le concept d'amour chez Augustin, Paris, Deux temps Tierce, 1991.

21. H. ARENDT, La vie de l'esprit 2 : Le vouloir, Paris, PUF, 1983, p. 123.

22. Sur le thème de l'amour augustin, voir Ronald BEINER, Love and Worldliness : Hannah Arendt's Reading of Saint Augustinen, dans Hannah Arendt Twenty Years Later, L. MAY et J. KOHN (éd.), Cambridge, MIT Press, 1996, pp. 269-284.

23. H. ARENDT, "Une situation de crise", dans Les Cahiers du GRIF, p. 147. Le titre est ajouté par la revue. 
pas sont comme des somnambules 24 ». Bref, on peut "dire que l'humanité vivante d'un homme décline dans la mesure où il renonce à la pensée25».

Fondée sur l'erôs socratique, le penser d'Arendt établit ainsi un lien avec l'amour-passion. Puisque en effet au niveau de l'esprit nous sommes toujours deux-en-un, l'actualisation du point de vue de l'autre dans la consciousness, autrui est ainsi toujours présent dans mon domaine intérieur. De sorte - nous semble-t-il, que la solitude - la dualité inhérente à l'activité de penser socratique - représente la condition à l'expérience de l'amour intime chez Arendt. II faut préciser qu'elle fait la distinction entre la solitude et "l'esseulement". La première se distingue du second dans la mesure où je suis, au sein de ce dernier, «incapable de me tenir compagnie. Je suis un et sans personne ${ }^{26}$ ". La solitude est conditionnée par la pluralité, ce qui n'est pas le cas de l'esseulement27. Qu'on nous comprenne bien. Arendt ne dit pas qu'il s'agit d'être animé du deux-en-un pour aimer une personne. Elle ne réduit pas l'amour à la mise en oeuvre de l'activité de penser. Elle dit simplement que l'individu esseulé, dénué du deux-en-un, du pouvoir de l'imagination, ne vit pas avec les autres dans le domaine de la pensée. II est donc moins en mesure de penser à autrui et donc d'aimer autrui.

Ce rapport entre moi et moi-même dans lequel je fais l'épreuve de la pluralité irréductible à l'unité, nous rappelle à un certain point la conception de l'amour chez Lévinas. Pour ce dernier, l'amour désigne dans la relation entre Je et Tu l'altérité radicale du visage d'autrui : "Je pense que quand autrui est «toujours autre", c'est là le fond de l'amour28". Lévinas ne voit pas dans le regard d'autrui, le Même, le Semblable, mais précisément l'Autre. II est ainsi réfractaire à toute dimension de fusion, d'unité ou de coincidence dans la relation amoureuse ${ }^{29}$. Chez Arendt, ce caractère d'altérité se rencontre non seulement dans sa reprise de l'erôs socratique - je désire ce que je ne suis pas, ce dont je manque -, mais aussi dans son souci de préserver le caractère distinct de l'individu30. L'amour, rappelons-le, révèle l'identité, le "qui» du sujet amoureux. Par là, elle

24. H. ARENDT, La vie de l'esprit 1 : La pensée, p. 217. Trad. mod.

25. H. ARENDT, "De l'humanité dans de «sombres temps", dans Vies politiques, Paris, Gallimard, 1974, p. 19.

26. H. ARENDT, La vie de l'esprit 1 : La pensée, p. 210.

27. H. ARENDT, "Compréhension et politique", dans La nature du totalitarisme, Paris, Payot, 1990, p. 126.

28. F. POIRIÉ, Emmanuel Lévinas. Essai et entretiens, Paris, Actes Sud, 1996, p. 124.

29. E. LÉVINAS, Le Temps et l'autre, Paris, PUF, 1983, p. 89. Voir également “De la phénoménologie à l'éthique. Entretien avec Emmanuel Lévinas», dans Esprit, no 234 (juillet 1997), p. 130.

30. H. ARENDT, "Travail, oeuvre, action », dans Études phénoménologiques, vol. 1, no 2 (1985), p. 21. Voir aussi H. ARENDT, Condition de l'homme modeme, p. 232. 
rejoint Lévinas. Elle le rejoint également sur un autre point. Dans le visage de l'Autre, le philosophe français y voit l'Infini. Rencontrer autrui, c'est sortir de la totalité, de l'être de l'étant et s'ouvrir à l'Infini. Or, l'erôs socratique, nous l'avons vu, ne fait pas fondre deux êtres en un seul; il est toujours cette quête infinie. Par l'amour socratique, Arendt n'est pas seulement confrontée à l'altérité de l'Autre, mais poursuit cette quête infinie de la compréhension de l'Autre et du monde. Ouverture à la distinction et à l'infini, ce rapprochement entre la philosophie morale d'Arendt et la pensée éthique de Lévinas n'occulte pas leurs différences. En premier lieu, elle ne conçoit pas l'amour comme une "responsabilité pour autrui». En deuxième lieu, elle ne pense pas la catégorie de l'amour socratique à l'épreuve d'une ontologie de l'être de l'étant; l'erôs ne constitue pas la réponse d'Arendt à une sortie de l'Être, alors qu'il est clair que Lévinas fonde sa théorie de la responsabilité pour autrui comme une réplique à l'ontologie de la totalité. Arendt pose certes la question qui fut celle de Leibnitz, de Schelling, de Heidegger et de Lévinas : «Pourquoi y-a-t-il quelque chose plutôt que rien?31", mais elle ne s'est pas interrogée sur la teneur de ce «il y a». En revanche, Lévinas a réfléchi à ce mot : celui-ci correspond non pas au "néant» heideggérien, mais plutôt à l'anonymat, à l'impersonne|32. Dans ce contexte, la responsabilité pour autrui représente bien une tentative pour sortir de l'Être. En d'autres termes, Arendt ne pense pas la question de l'erôs sur le plan de l'être de l'étant, tandis que c'est sur ce plan que Lévinas fonde son éthique. Au demeurant, l'importance accordée par elle non seulement au caractère distinct de l'homme, mais également à l'ouverture pour autrui annonce les analyses de Lévinas sur le thème de l'amour.

\section{Erôs et politique}

Animée de l'amour socratique, Arendt retrouve aussi ce type d'amour dans lequel, d'une part, j'entreprends un dialogue avec moi-même - un ami - et dans lequel, d'autre part, je ne fusionne jamais avec l'objet aimé, au sein de la poésie de Wystan Hugh Auden. Dans l'article qu'elle lui consacre ${ }^{33}$, elle reprend le jugement formulé par l'ami d'Auden, Stephen Spender : «ll était frappé que «durant tout le développement de la poésie de Auden... son thème était l'amour (cela engageait Auden à changer le "cogito ergo sum" de Descartes en définissant l'homme comme une

31. H. ARENDT, Qu'est-ce que la politique?, Paris, Seuil, 1995, p. 139.

32. E. LÉVINAS, Éthique et infini. Dialogues avec Philippe Nemo, Paris, Fayard, 1982, p. 37.

33. H. ARENDT, «Reflections : Remembering Wystan H. Auden... ", dans The New Yorker (20 janvier 1975), pp. 39-46. 
"créature avec une tête bouillonnante» (bubble-brained creature) et à dire "je suis aimé donc je suis ${ }^{34}$ ». Arendt ajoute : "À la fin de l'allocution que Spender donnait en mémoire de son vieil ami, il parla d'un discours que Auden avait donné en Amérique. "Le visage [de Auden] se redressa avec un sourire qui altéra sa lecture, et il dit : «ils m'aiment». Ils ne l'admiraient pas, ils l'aimaient«. Elle voit donc "Auden comme un expert dans les variétés infinies de l'amour, parmi lesquelles la substitution de l'admiration pour l'amour doit sûrement apparaître très importante «. L'amour a ici une dimension publique dans le sens où il se substitue à l'admiration. L'amour du public pour Auden surpasse l'admiration du public pour sa poésie. Cet amour implique non seulement le respect entre le poète et le public, mais il implique aussi l'amitié entre ces deux derniers. L'amour maintient ainsi une distance, un espace entre le premier et le second dans lequel le monde s'insère. Auden révèle ainsi son identité au sein de ce rapport amoureux : "Je suis aimé donc je suis". En faisant un usage public, pour utiliser une expression kantienne, de sa poésie, il a révélé son "qui». Pour Arendt, Auden rejoint ici sa conception de l'amour-passion : il dévoile l'identité de l'être aimé et il dégage le caractère politique de l'amour. L'amour établit en quelque sorte un dialogue politique entre le poète et ses auditeurs. II est donc d'une certaine façon une condition à la fondation de l'être ensemble.

On peut établir un autre lien entre l'erôs et la politique en examinant l'analyse que fait Arendt, en collaboration avec son premier mari, Günther Stern, du concept de l'amour rilkéen dans leur texte sur les Élégies de Duino35. Arendt et Stern ne procèdent pas, dans leur article, à une analyse systématique et intégrale du poème de Rainer Maria Rilke. Les deux auteurs préfèrent étudier certains vers tirés des dix Élégies de ce dernier. Comment exploite-t-il, selon les deux écrivains politiques, le thème de l'amour dans son poème? À l'égard de ce thème, les deux philosophes analysent plus particulièrement quelques vers de la première Élégie de Rilke. Ces vers sont les suivants : "L'as-tu donc célébrée assezl la mémoire de Gaspara Stampa, pour que la jeune femme à qui échappa son amant, devant l'exemple immense d'une telle amante, pût s'émouvoir et souhaiter: Je voudrais être comme elle/ Et ces douleurs plus anciennes, ne faut-il pas qu'elles nous deviennent plus fécondes enfin?/ N'est-il pas temps que nous nous libérions, en l'aimant, de l'aimé, et que, vibrants nous lui résistions : ainsi la flèche résistant à la corde de l'arc, sa force toute concentrée dans l'élan, pour devenir plus qu'elle-

34. Ibid., p. 40.

35. H. ARENDT et STERN, «Les élégies de Duino", dans Poésie, no 38 (1986), pp. 37 52. 
mêmes6». Commentant ces vers, Arendt et Stern concluent : "L'amour [chez Rilke] est d'autant plus amour qu'il est moins assouvi». Cette phrase fait, bien sûr, penser aux mots de Socrate : l'amour est toujours le désir d'une chose qui nous manque. Il est constamment cette tension vers l'être désiré. Chez Rilke, ce manque se révèle précisément, selon les deux philosophes, dans le vers suivant: "Hélas! ils se voilent seulement l'un à l'autre leur destin's. Pour eux, ce vers signifie que les amoureux sont maintenus à distance de manière irréductible. L'amour rilkéen est donc similaire ici à l'erôs socratique : ces deux types d'amour séparent l'être plutôt qu'ils ne l'unissent. Le premier rejoint également le second, car il établit aussi un rapport au monde, un rapport entre le sensible et l'invisible : "L'amour [rilkéen] dépasse et oublie tout à la fois la personne aimée, puisqu'il a en vue plus que lindividu fortuit et que la proximité de l'amant déplace son horizon. II n'y a d'amour que dans cet abandon; détaché de l'amant, l'amour, libéré dans l'espace de son propre horizon peut devenir l'organon pour la compréhension des rapports au monde37". L'amour rilkéen est plus ici qu'un amour non assouvi, il est un mode de pensée ou un processus de pensée qui trace un lien entre le sensoriel et l'intellectuel, rend compréhensible et significatif pour la pensée le monde phénoménal. L'amour dépasse ici sa "compétence immanente pour ouvrir le regard sur les rapports au monde ${ }^{38}$ ". II est ici non seulement le "pré-réquisit» à l'activité de penser, mais il ouvre aussi la voie à la compréhension du monde. L'amour rilkéen rejoint ici l'amour socratique. Le premier rappelle ici le second dans la mesure où tous les deux sont tournés non pas vers le domaine privé mais vers le monde. Ils établissent tous deux un rapport au monde : Socrate tente de saisir le sens des concepts originaux, tandis que Rilke veut aller au-delà de l'amour entre deux êtres, il tente de comprendre le monde phénoménal. Processus réflexif de compréhension, l'amour rilkéen et l'amour socratique sont également tournés vers la réalité, le monde commun.

Si l'amour est plus pour Arendt qu'un désir inassouvi, mais aussi un mode de compréhension du monde humain, alors il faut ici se tourner vers Heidegger qui constitue le second apport majeur à la conception arendtienne de la vie de l'esprit. Pour notre propos, il faut retenir ici de l'apport heideggérien à l'activité de penser d'Arendt deux éléments : 1) penser consiste à entreprendre un chemin de réflexion et 2) penser consiste aussi à comprendre et non pas à connaître les objets aimables. Pour la phénoménologue, le processus de pensée heideggérien se réalise en

36. Ibid., p. 46, nous soulignons.

37. Ibid., p. 44, c'est nous qui soulignons.

38. Ibid., p. 46. 
entreprenant un chemin de pensée. Ce processus ne s'effectue, en effet, "qu'en chemin, le long du chemin 39 ". Le penser de Heidegger pose des jalons qui, balisant le chemin, oriente un nouveau cours de penser ${ }^{40}$. Ainsi, la quête de Socrate, c'est au fond pour Arendt un chemin de pensée heideggérien. Bien entendu, ce chemin de pensée qui est simultanément socratique et heideggérien chez Arendt, n'est pas le «chemin de campagne" de Heidegger. L'activité de penser de la philosophe entreprend un chemin qui n'est pas un chemin "à travers [les] champs". Son chemin, c'est celui - nous l'avons vu - de l'amour du thaumadzein. Plus important : elle reprend surtout de Heidegger la fameuse dichotomie qu'il fait entre "un objet savant [et] une chose pensée 41 ", entre la connaissance et la pensée. Pour Arendt, l'activité de penser n'est en effet "mis[e] en mouvement ni par la soif de savoir ni par le besoin de connaissance ${ }^{42}$ ", mais elle est plutôt mise en mouvement par le besoin de compréhension. Plus précisément, elle établit une typologie de la compréhension. Il y a d'abord la compréhension "préliminaire" qui constitue le fondement de la connaissance. Puis, il y a la "véritable compréhension" qui "opère un dépassement par rapport à cette connaissance ${ }^{43}$ ". Pour elle, ces deux types de compréhension partagent un point commun : elles donnent un sens à la connaissance. Mais le point important à souligner ici, c'est que la compréhension produit du sens. La compréhension nous réconcilie avec la réalité, le monde réel. La quête de sens de l'activité arendtienne de penser ne consiste pas seulement à déconstruire la connaissance pour saisir le sens originel des concepts, elle consiste surtout à comprendre le monde. Nous sommes ici en mesure de comprendre la thèse défendue par Arendt, à savoir "l'amour peut devenir l'organon de la compréhension des rapports au monde". L'amour du questionnement la motive non seulement à poursuivre une quête de sens des objets de pensée, mais la conduit aussi à comprendre les événements politiques constitutifs de l'être ensemble des hommes. L'activité de penser d'Arendt n'épouse donc pas seulement les caractères du penser de Socrate, elle épouse également certains caractères du penser de Heidegger ${ }^{44}$.

39. M. HEIDEGGER, Le principe de raison, Paris, Gallimard, 1962, p. 146.

40. H. ARENDT, «Heidegger a quatre-vingts ans", dans Vies politiques, p. 311.

41. M. HEIDEGGER, "L'Expérience de la pensée», dans Questions III, Paris, Gallimard, 1966, p. 23. Voir aussi M. HEIDEGGER, Qu'appelle-t-on penser?, Paris, PUF, 1959, p. 243.

42. H. ARENDT, «Heidegger a quatre-vingts ans", p. 312.

43. H. ARENDT, «Compréhension et politique" La nature du totalitarisme, p. 43.

44. Pour une analyse plus fine de la dette d'Arendt à l'égard du penser de Heidegger, voir l'ouvrage de J. TAMINIAUX, La fille de Thrace et le penseur professionnel : Arendt et Heidegger, Paris, Payot, 1992. 
Sur le rapport entre le concept de l'amour et la vie de l'esprit, il est intéressant de comparer la thèse défendue par Arendt - l'amour est la condition à l'activité de penser - à celle de Heidegger. Dans son livre Qu'appelle-t-on penser?, le philosophe allemand cite d'abord deux strophes d'un poème de Holderlin, Socrate et Alcibiade : "Socrate Sain et Sauf/ Pourquoi rendre ce culte/ Sans cesse à la jeunesse/ De celui-ci/ $\mathrm{Ne}$ sais-tu rien qui soit plus grand ?/ Pourquoi complaire ton regard/ Qui paraît voir les Dieux/ Et celui-ci». Seconde strophe : "Qui a pensé le plus profond aime le plus vivant Et comprend la haute jeunesse/ Qui a percé le monde à jour/ La fin des Sages penche/ Souvent au-dessus du beau45". Puis, il analyse très brièvement et plus particulièrement un vers du poème de Holderlin : "Qui a pensé le plus profond aime le plus vivant». Pour Heidegger, ce vers signifie que Socrate «fonde l'amour sur la pensée». L'amour repose, autrement dit, dans l'acte de penser. L'activité de penser socratique est donc en quelque sorte la condition pour aimer. Heidegger n'en dit toutefois pas davantage sur ce rapport entre l'erôs socratique et l'acte de penser. (II n'en dit pas plus dans son texte "Que veut dire penser?"). En revanche, nous avons vu que l'amour est pour Arendt la condition à l'acte de penser. Sur ce point, les deux philosophes divergent radicalement. Pour la professeure de théorie politique, l'amour conditionne la pensée, tandis que c'est la pensée qui, au contraire, conditionne l'amour chez le phénoménologue. En outre, et il est important de le souligner : elle est tout à fait favorable à lidée de fonder l'activité de penser sur l'amour; Heidegger, lui, dénonce lidée de fonder la raison sur l'amour ${ }^{46}$. Sur le rapport entre l'erôs socratique et le penser, Arendt et Heidegger ne s'entendent donc pas du tout. Cette divergence se reflète dans les concepts constitutifs de la pensée d'Arendt et de la pensée de Heidegger. Alors que la première fonde sa théorie politique sur une philosophie de la natalité dans laquelle le nouveau venu renferme en lui un potentiel de nouveauté susceptible de transformer le monde pour le mieux;

projet du second qui consiste à dépasser l'ontologie occidentale - l'être de l'étant - s'effectue en se tournant vers «l'être-pour-la-mort". Or, la mort représente limpossibilité de fonder un projet politique chez Arendt; elle met un terme à l'action de façon concertée. D'un côté, nous avons une pensée qui tente d'établir un monde commun par amour de la condition humaine, tandis que de l'autre côté, on est en présence d'une pensée

45. M. HEIDEGGER, Qu'appelle-t-on penser?, p. 32. Nous soulignons. Heidegger reproduit aussi ces deux mêmes strophes du poème de Holderlin dans son texte "Que veut dire penser?", dans Essais et conférences, Paris, Gallimard, 1966, p. 163. 
qui, confrontée à la mort - épreuve ultime du solipsisme du Dasein par laquelle l'individu s'arrache à la banalité de la vie quotidienne, à l'anonymat du "on" -, trouve ainsi sa voie vers le chemin de la "sortie» de l'ontologie. Autrement dit, Arendt pose les conditions fondamentales avec lesquelles les hommes ont affaire à la politique; Heidegger pose, pour sa part, la condition essentielle pour renouer avec l'Étre : je suis voué à la mort, «je suis à la mort». Lévinas avait d'ailleurs ce mot tout à fait juste pour décrire le projet de Heidegger : «ll y a chez lui le rêve de noblesse du sang et de l'épée 47 ". Bref, Arendt pense la condition humaine par amour pour le monde, Heidegger pense, lui, la mort comme la possibilité la plus authentique du Dasein.

Enfin, nous avons spécifié au début de cet article que l'erôs de Socrate met en oeuvre la liberté de penser arendtienne. En quel sens l'activité de penser est-elle libre chez elle? Précisons d'abord que pour Arendt la liberté ne coincide pas seulement en effet avec l'action, mais également avec la pensée : "La liberté réside dans les aptitudes de l'homme à agir et à penser 48 ". Le penser arendtien est dit libre dans la mesure où il repose, d'une part, sur les caractères de l'activité de penser de Socrate et, d'autre part, sur les deux caractères de l'activité de penser de Heidegger. En d'autres termes, si l'activité de penser n'est pas conditionnée par l'erôs socratique (la quête de sens ou le chemin de penser chez Heidegger), le deux-en-un, ne rejette pas la contradiction et ne vise pas à connaître les phénomènes, mais plutôt à les comprendre, cette activité de penser n'est dite pas libre. Cependant, la condition primordiale à l'exercice de penser demeure pour Arendt l'amour socratique, car c'est ce désir amoureux - je désire quelque chose que je ne possède pas - qui met en mouvement la pensée, la "projette" sur le chemin de la quête infinie de sens. L'amour de la liberté de penser, c'est donc l'amour de l'activité de penser dans son effort de comprendre le point de vue de l'autre et donc de se le représenter. Et c'est aussi l'effort de comprendre, d'une part, le sens étymologique des concepts et l'effort de comprendre, d'autre part, les événements politiques dans lesquels se révèlent les conditions de possibilité de l'action politique. L'amour de la sagesse, la quête de sens, nous invite à penser l'être-dans-le-monde.

\section{L'amour de la liberté politique}

L'amour n'est pas seulement pour Arendt ce qui met en mouvement le processus de pensée dans son effort de comprendre le sens d'un objet

46. M. HEIDEGGER, Qu'appelle-t-on penser?, p. 33.

47. E. LÉVINAS, Les Imprévus de l'histoire, Paris, Le Livre de Poche, 2000, p. 186.

48. H. ARENDT, “Epilogue : Reflections on the Hungarian Revolution", dans The Origins of Totalitarianism, Cleveland, Meridan Book, 1958, p. 502. 
de pensée, il est aussi la condition en quelque sorte d'une seconde vie, c'est-à-dire une vie politique. La réflexion de la phénoménologue s'inspire grandement d'un vers qu'elle puise, semble-t-il, dans un des poèmes de Goethe, Amyntas : "Celui qui a confiance dans l'amour, délibère-t-il au sujet de sa vie?» Elle tient à souligner ici que celui qui est animé par l'amour de la liberté politique s'est libéré de la nécessité, de la question sociale; il est donc apte à exercer l'agir de concert : "Le risque qui doit être exigé de celui qui se consacre à la sphère du politique, où il doit délibérer de tout plutôt que de sa vie, ne concerne nullement, aussi longtemps du moins que tout se déroule normalement, la vie de la société, de la nation ou du peuple, à laquelle il aurait dû sacrifier la sienne. Le risque concerne bien plutôt exclusivement la liberté, la sienne comme celle du groupe auquel l'individu peut appartenir...49». C'est donc l'amour de la liberté qui nous dégage des nécessités de la vie. On pourrait compléter le vers de Goethe et dire que chez elle, celui qui a confiance dans l'amour de la liberté, délibère-t-il au sujet de sa vie? En étant amoureux de la liberté, l'homme est alors en mesure de pouvoir agir avec ses pairs. En aimant la liberté politique, l'homme cesse d'être un homme socialisé, il peut devenir un citoyen exerçant le politique. Pour bien mesurer le propos d'Arendt, il importe de savoir ici ce qui constitue pour elle une question sociale. Celle-ci est une question qui peut être résolue selon des calculs arithmétiques; la question sociale relève de «l'administration des choses». En revanche, une question politique est pour elle une question "ultime", comme, par exemple, qu'est-ce que l'autorité, qu'estce que l'éducation, qu'est-ce que la justice?, etc. La question politique demeure donc toujours ouverte, ne peut relever de l'administration des choses. Lors d'un colloque à Toronto portant sur son oeuvre, elle s'était expliquée sur la distinction qu'elle faisait entre la question sociale et la question politique : "ll y a des choses où les mesures exactes peuvent être établies. Ces choses peuvent être vraiment administrées et ne sont pas alors sujets à des débats publics. Le débat public peut seulement traiter des choses que - si nous voulons le dire négativement - nous ne pouvons pas mesurer avec certitude. Autrement, si nous pouvions les mesurer avec certitude, pourquoi aurions-nous besoin d'établir un monde commun?50". Or, quelle est l'organisation sociale dont la fonction est pour la philosophe d'administrer ces choses? La bureaucratie étatique, répond-elle. Le rôle social de l'État consiste ainsi, d'une part, à pourvoir

49. H. ARENDT, Qu'est-ce que la politique?, p. 84. Souligné par nous. Selon l'éditrice de l'ouvrage d'Arendt, Ursula Ludz, le mot que nous soulignons «est peut être inspirén du vers de Goethe.

50. Voir " Hannah Arendt on Hannah Arendt ", dans Hannah Arendt : The Recovery of the Public World, New York, St. Martin's Press, 1979, p. 317. 
aux nécessités de la vie et il consiste, d'autre part, à protéger la liberté sociale, c'est-à-dire la libre expansion «des forces productives de la société». Pour Arendt, il ne fait pas de doute que ce rôle social est fortement dominant au sein de l'État. Dans ce contexte, le rôle politique de l'État est dès lors, pour le moins, problématique. Le chef du gouvernement, conseillé par des experts, prend des décisions politiques dans lesquelles l'esprit public est sensé s'exprimer. Or, ce phénomène est précisément "hautement mystérieux»51. En d'autres termes, la domination de la liberté sociale entraîne un affaiblissement du débat public dans la modernité. L'amour de la liberté politique constitue pour Arendt le "remède" à ce problème : il invite les hommes à outrepasser la question sociale pour les inciter non seulement à se poser des questions politiques mais aussi à agir politiquement. Le point est évidemment crucial dans la pensée politique de la théoricienne. C'est en étant amoureux de la liberté que nous serons en mesure de participer à l'action politique, de prendre des décisions politiques. C'est l'amour de la liberté qui peut faire de nous un peuple politique. II est la condition à l'exercice de l'action chez Arendt.

On retrouve aussi ce thème de l'amour de la liberté dans l'analyse qu'elle fait de ses fameuses fondations légendaires. Pour elle, c'est l'amour, d'une part, de la libération (première étape de l'action politique) et c'est l'amour, d'autre part, de la liberté d'agir (seconde étape de l'action politique) ${ }^{52}$ qui balisent le processus historique de l'agir dans lequel les hommes sont conviés à exercer la liberté politique : "Les deux fondations légendaires de la civilisation occidentale, l'une romaine, l'autre hébraïque (...) attestent que dans la fondation - acte suprême au cours duquel le "Nous" se constitue en entité identifiable - le principe d'inspiration de l'action est l'amour [love] de la liberté au sens négatif de libération de l'oppression, et au sens positif d'établissement d'une Liberté comprise comme réalité stable et tangible ${ }^{53}$ ". Ces légendes fondatrices ne sont pas des récits mythiques, "merveilleusement colorés", elles "parlent (au contraire) toujours de faits concrets ${ }^{54}$ ». Ils décrivent la réalité de l'expérience politique ancestrale des Juifs - l'exil (Moïse) - et la réalité de la fondation de Rome par Enée. Pour Arendt, ces légendes décrivent des expériences réelles de liberté politique. C'est pourquoi elle prend au sérieux ces légendes. Elle ne retrouve pas seulement ce principe d'inspiration de l'action dans les récits légendaires, mais aussi non seulement dans la vie

51. H. ARENDT, «Seule demeure la langue maternelle», p. 253.

52. H. ARENDT, «La guerre froide et l'Occident", dans Penser l'événement, Paris, Belin, 1989, p. 204.

53. H. ARENDT, La vie de l'esprit 2 : Le vouloir, p. 232-233.

54. H. ARENDT, L'impérialisme, Paris, Seuil, 1984, p. 148. 
politique des Romains - la res publica - et également dans l'expérience grecque de la polis. Dans le monde moderne, elle retrace ce même principe dans plusieurs expériences politiques comme la Révolution américaine, la Révolution française, la Commune de Paris, les Révolutions russes, la République des conseils et la Révolution hongroise. Pour la phénoménologue, l'action est donc toujours mise en oeuvre par un principe, c'est-à-dire l'agir est toujours précédé d'un principe de conviction partagé par les acteurs qui les conduit à agir ensemble. Notons ici qu'elle n'entend pas seulement la notion de principe dans le sens de Montesquieu. Elle s'inspire également, nous semble-t-il, de la pensée kantienne. Quand elle affirme : si l'on pouvait découvrir que dans les échanges entre "des hommes étroitement liés les uns aux autres par la commune possession d'un monde, il existe un principe a priori, alors on aurait la preuve que l'homme est un être essentiellement politique 55 ", nous croyons qu'elle puise le sens de cette notion d'a priori au sein de la théorie kantienne dans la mesure où ce principe a priori n'est certes pas pour Arendt une condition de la connaissance, mais plutôt une condition universelle de l'action politique. II s'agit de trouver un principe a priori, c'est-à-dire qui précède l'expérience et donc universel, pouvant démontrer que les hommes agissent ensemble. N'empêche que cette appropriation arendtienne du principe a priori kantien diffère néanmoins de la signification du concept de principe chez Montesquieu. Comment peut-elle donc associer les pensées de ces deux auteurs? Pour Arendt, le principe de l'action est universel (Kant), tandis que son contenu est particulier (Montesquieu). Le principe de l'action est valide pour l'ensemble des acteurs politiques, mais il s'actualise toujours dans une expérience unique et singulière du politique. Or, le principe par excellence de l'action, c'est celui de l'amour de la liberté56. Cet amour de la liberté représente la condition effective de la participation des hommes au maniement des affaires humaines. Sans cet amour de la liberté, il n'y aurait point de possibilité d'expérience de l'action arendtienne. II est non seulement le principe le plus important qui préside à la fondation politique, mais il est aussi ce qui incite les acteurs à se libérer de l'oppression et à fonder, dès lors, un corps politique dans lequel s'exerce la liberté d'agir. Arendt puise donc la source de sa théorie politique au sein de cet amour. II est la matrice du politique arendtien.

L'amour a aussi un rapport au temps chez elle. L'amour de la liberté occupe, en effet, dans le texte mentionné plus haut, l'espace de l'hiatus

55. H. ARENDT, Juger. Sur la philosophie politique de Kant, Paris, Seuil, 1991, p. 196.

56. H. ARENDT, "La nature du totalitarisme. Essai sur la compréhension», dans La nature du totalitarisme, pp. 68 et 71. 
entre ce qui n'est plus et ce qui n'est pas encore, "entre le désastre et le salut, (ou encore) entre l'instant où l'on secoue l'ordre ancien et la liberté neuve incarnée en un novus ordo sæculorum, un nouvel âge du temps57". L'amour de la liberté est, d'une part, la condition pour commencer quelque chose de nouveau, mais il est aussi, d'autre part, ce qui brise le "flux continu de l'enchaînement temporel». Poser la question du rapport entre la liberté et le temps, c'est poser au fond la question de la nouveauté chez Arendt. Qu'est-ce qu'une «liberté neuve»? On trouve, il nous semble, la meilleure "définition" de ce mot dans son ouvrage Crises of the Republic: "Un des traits marquants de l'action humaine est qu'elle entreprend toujours du nouveau, ce qui ne signifie pas qu'elle puisse alors partir de rien (to start ab ovo), créer à partir du néant (create ex nihilo). On ne peut faire place à une action nouvelle qu'à partir du déplacement (remove) ou de la destruction de ce qui préexistait et de la modification (change) de l'état de chose existant58". L'avènement de la nouveauté suit donc deux étapes chez Arendt : 1) l'anéantissement de l'ancien et 2) le changement de ce qui est. La destruction ne signifie pas qu'on fait tabula rasa du passé. La nouvelle création n'est pas ex nihilo. Le passé est toujours présent et actualisé dans la mémoire des acteurs politiques agissant. Elle écrit : «À l'évidence, l'aptitude au changement n'est pas plus illimitée chez l'homme que sa capacité de préservation, la première étant réduite par l'influence du passé sur le présent - aucun homme ne commence $a b$ ovo - et la seconde par le caractère imprévisible de l'avenir59". Cependant, ce passé n'est d'aucun secours pour les acteurs politiques, car ceux-ci doivent précisément non pas réformer l'ancien gouvernement, mais fonder un nouveau gouvernement. Tout se passe comme si les acteurs en brisant le cours linéaire de l'histoire, il ne leur reste alors plus «rien à quoi se tenir». La nouvelle fondation de la liberté politique cristallise ainsi des fragments du passé transformés par les acteurs qui leur donnent une nouvelle configuration politique. La liberté est nouvelle dans la mesure où le gouvernement n'a jamais "envahi ni dirigé le maniement des affaires humaines 60 ". C'est donc la "tonalité générale» de la nouvelle fondation politique qui confère sa dimension nouvelle à la liberté. Par l'expression "ex nihilo», Arendt souligne ainsi que l'histoire est ponctuée de ruptures, de brèches; qu'elle est donc discontinuée. Par là, elle rejette la philosophie de l'histoire hégélienne dans le sens où elle tente de repenser le temps et donc le principe de raison en le soustrayant au processus rationnel et nécessaire de l'histoire. L'amour de la liberté

57. H. ARENDT, La vie de l'esprit 2 : Le vouloir, p. 234.

58. H. ARENDT, Du mensonge à la violence, Paris, Calmann-Lévy, 1972, p. 9.

59. bid., p. 80.

60. H. ARENDT, Le système totalitaire, Paris, Seuil, 1972, p. 204. 
constitue non seulement le principe par lequel les hommes se libèrent de la nécessité, puis décident d'agir de concert pour fonder la nouvelle liberté, mais il constitue aussi le moment de rupture historique chez Arendt. L'amour de la liberté est la quintessence des principes qui institue la brèche historique.

L'amour de la liberté est si fort chez elle que même un régime totalitaire ne parvient pas à ul'extirper du cœur des hommes 61 ». Précisons d'abord que ce cœur est politique. II n'existe en effet qu'en relation avec le monde : "Le cœur humain, aussi éloigné de la sensiblerie que de l'activité routinière, est la seule chose au monde qui puisse assumer le fardeau que nous a légué le don divin de l'action, ce don d'être un commencement et, partant, d'être capable de commencer62». C'est dire que seuls les hommes qui possèdent un "cœur compréhensif63" sont en mesure d'agir avec les autres. En associant ce "cœur compréhensif» à l'amour, Arendt fait d'une certaine façon de ce dernier la condition de l'agir politique. Dans ce sens, un régime totalitaire ne parvient pas à extirper la liberté du cœur des hommes parce que nous sommes, pourrions-nous dire, "condamnés à la liberté". Cette formule peut prêter à confusion : Arendt ne dit pas que la liberté d'action est le fait de la nature humaine, de la natalité, elle dit que la liberté est une réponse au don de l'action. L'homme n'est pas seulement un commencement, il est aussi celui qui commence ${ }^{64}$. Nous sommes donc condamnés à agir, car c'est par l'action concertée que nous établissons un monde. On touche ici le cœur de la conception arendtienne de l'idée d'une humanité politique. La fondation d'un monde commun est une invention dans la mesure où ce sont l'action et la fabrication qui l'engendrent. L'action engendre un monde par l'exercice, d'une part, de l'agir à plusieurs et par l'exercice, d'autre part, de la parole dans un dialogue pluriel. La fabrication crée un monde d'objets durable, condition à l'action humaine. L'homme est l'acteur et le producteur du vivre-ensemble. Parce que l'homme est toujours déjà dans un

61. bid., p. 212. Nous soulignons.

62. H. ARENDT, "Compréhension et politique", dans La nature du totalitarisme, p. 59.

63. Nous reprenons ici la traduction française de M. Brenas, J. Bureau et A. Enegrén, "Compréhension et politique", dans Esprit, no 1667 (1985), p. 100. Nous n'avons pas repris la version de Launay qui, dans sa traduction de ce même texte d'Arendt, "Compréhension et politique", dans La nature du totalitarisme, suggère plutót la formule un "coeur intelligent" (p. 59). Or, il nous semble important de faire la distinction entre l'intelligence et la compréhension chez Arendt. Nous avons vu que la première relève de la connaissance, tandis que la seconde relève de la pensée. La compréhension est "véritablement une fonction de l'esprit, mais jamais le résultat automatique de l'intelligence". "La conquête de l'espace et la dimension de l'homme", dans La crise de la culture, p. 342.

64. H. ARENDT, "Qu'est-ce que la liberté ?", dans La crise de la culture, p. 222. 
monde qui le précède, la liberté d'agir défie précisément cette condition humaine d'être jeté dans le monde. Nous sommes également condamnés à agir parce que «la liberté de penser et d'action n'est possible que dans le cadre d'un savoir incertain et limité65", c'est-à-dire nous sommes des êtres libres, car nous ne maîtrisons pas la destinée humaine. Si nous connaissions parfaitement les hommes, nous serions alors en mesure de maîtriser leurs agissements. Nous sommes libres parce que les hommes ne sont pas complètement transparents à eux-mêmes. La liberté politique n'est possible que dans un monde où l'action est imprévisible. (C'est pourquoi, l'agir politique a un aspect tragique chez Arendt). Dans cet habitat commun institué historiquement et politiquement, nous sommes donc en quelque sorte forcés de nous créer nous-mêmes «dans un océan de possibilités chaotiques". C'est donc l'expérience de la liberté qui engendre non seulement l'égalité politique, mais aussi l'identité du sujet agissant. L'égalité résulte non pas d'un droit naturel, mais de l'expérience des acteurs. Arendt ne défend pas une conception libérale de la liberté; elle soutient davantage une conception "anarchique 66 » de la liberté. L'amour de la liberté politique est, en définitive, la condition pour organiser "d'emblée des êtres absolument différents en considérant leur égalité relative et en faisant abstraction de leur diversité relative67".

On est également en mesure de saisir une autre de ces formules quelque peu énigmatiques chez la philosophe. Dans le "troisième" volume sur La vie de l'esprit, Le juger, elle met en exergue ces vers de Goethe tirés de Faust : "Que ne puis-je Magie, t'éloigner de ma route/ Je voudrais désapprendre tous les sortilèges / $\mathrm{Si}$ je pouvais, Nature, face à toi, n'être qu'un homme seul/ L'humaine condition vaudrait alors la peine ${ }^{68}$ \%. Mais il importe de souligner ici qu'il y a un autre vers dans le poème dramatique de Goethe qui précède immédiatement cette épigraphe et qui mérite notre attention. Ce vers dit ceci : "Je n'ai pas encore trouvé mon chemin vers la liberté 69 \%. Ne parvenant pas à connaître, mais seulement à comprendre ce que signifie le concept de liberté, Arendt ne pouvait pas, bien entendu, découvrir son chemin vers la liberté.

65. H. ARENDT, «Religion et politique», dans La nature du totalitarisme, p. 124.

66. On entend ce mot dans le sens que lui donne Reiner Schürmann : "L'anarchie... est le nom pour une histoire qui a affecté le fondement de l'agir, histoire où cèdent les assises et où l'on s'aperçoit que le principe de cohésion, qu'il soit autoritaire ou rationnel, n'est plus qu'un espace blanc sans pouvoir législateur sur la vie." $L \theta$ Principe d'anarchie: Heidegger et la question de l'agir, Paris, Seuil, 1982, p. 16.

67. H. ARENDT, Qu'est-ce que la politique?, p. 34.

68. H. ARENDT, Lectures on Kant's Political Philosophy, Chicago, University of Chicago Press, 1982, p. ii. Cet épigraphe n'a pas été repris dans la traduction française.

69. Cité dans H. ARENDT, Juger. Sur la philosophie politique de Kant, p. 177. 
Objet de pensée non pas connaissable mais seulement compréhensible, la liberté est un objet introuvable. C'est dire : nous sommes condamnés à agir ensemble tout en sachant fort bien que nous sommes incapables de prouver l'existence de la liberté. (Et c'est sans doute la raison pour laquelle d'aucuns soutiennent que la liberté n'existe pas). Au demeurant, "l'humaine condition" vaut la peine, car l'amour de la liberté est le principe qui rend effectif l'expérience du politique arendtien.

Reste un dernier point à examiner : si la liberté réside dans la faculté de penser et dans la capacité des hommes à agir ensemble, cela signifie que la pensée et l'action partagent un caractère commun, c'est-àdire celui de la pluralité. L'activité de penser et l'action sont conditionnées par la relation à autrui dans le sens où le deux-en-un conditionne le penser et dans le sens où les hommes agissent ensemble chez Arendt. C'est pourquoi le penser et l'action sont politiques. En soumettant la pensée à l'épreuve de la réalité et donc de l'altérité, Arendt fait l'expérience du politique au niveau de l'esprit. En agissant avec ses semblables, elle fait, bien entendu, l'expérience de la liberté politique. L'effort constant déployé par la professeure de théorie politique pour comprendre le monde souligne qu'elle concevait l'activité de penser comme une activité politique. Par là, elle tenait à répliquer, disait-elle, à la tradition philosophique fondée par Platon selon laquelle la pensée est une activité contemplative soustraite à l'épreuve du réel. On voit ainsi se dessiner le projet politique d'Arendt : fonder une nouvelle philosophie politique dans laquelle le penser confronte la raison à l'action. Mais cela est évidemment une autre question.

Au total, l'amour (erôs) fonde le processus de l'activité de penser chez Arendt, c'est-à-dire il met en mouvement le deux-en-un qui poursuit une quête de signification en vue de comprendre le sens original d'un objet de pensée. II poursuit cette quête parce que l'amour est toujours à la recherche de ce qui lui manque. L'amour n'est pas complétude, mais penser de l'absence. C'est l'amour de l'activité de penser qui la conduit à penser ce que nous faisons. Celui qui est animé de l'amour socratique pense le plus profond. D'autre part, l'amour (love) de la liberté politique, le principe énergétique par excellence à l'expérience de l'action, constitue non seulement la condition pour s'émanciper de la nécessité, de la question sociale, mais il représente aussi la condition pour fonder la vie politique chez Arendt. C'est en étant amoureux de la liberté politique que nous serons en mesure d'agir de façon concertée avec nos semblables. Cet amour de la liberté est également le principe qui établit le nouveau commencement; il est ainsi ce qui rompt le cours linéaire de 
I'histoire. Pour échapper à la nécessité historique, il faut se jeter dans l'action. L'amour de la liberté politique et l'amour de la liberté de penser fondent en définitive l'idée d'une humanité politique chez Arendt. II n'est pas inutile de se rappeler ces thèses arendtiennes car, à une époque où l'amour est exclusivement identifié à l'amour-passion, à l'amour intime, il est fécond de lire une auteure qui, refusant de réduire le politique aux bons sentiments, tente d'établir une politique fondée sur l'erôs.

\author{
Francis Moreault \\ Département de science politique \\ Université d'Ottawa
}

